\title{
Design of an All-Semiconductor Selective Metamaterial Emitter in the Mid-IR Regime with Larger Feature Sizes for Thermophotovoltaic Energy Conversion Applications
}

\author{
MINSU OH (i), ${ }^{1}$ EMILY CARLSON (i), ${ }^{1}$ and THOMAS E. VANDERVELDE (i) ${ }^{1,2}$ \\ 1.-Department of Electrical and Computer Engineering, Tufts University, \\ Medford, MA 02155, USA. 2.—e-mail: tvanderv@ece.tufts.edu
}

A thermophotovoltaic (TPV) system converts heat that is absorbed via conduction, convection, and/or radiation to electricity. The efficiency of TPV energy conversion can be improved with a narrowband selective emitter that emits photons at just above the bandgap energy towards the TPV photodiode. We numerically report a selective metamaterial (MM) emitter design with a single layer of cylindrical structures of $p$-type silicon (boron-doped). Our design (substrate-free) features a peak absorbance of $94.8 \%$ at the wavelength of $3.47 \mu \mathrm{m}$ with the smallest lateral dimension of $0.8 \mu \mathrm{m}$. The absorption is found to be due to the resonance of electric and magnetic fields in the structure. The larger dimensions of our selective MM emitter design make it significantly easier to pattern than many of previously reported selective MM emitters operating at similar wavelengths to that of our work. We believe that our work demonstrates a path forward for future research on larger-area all-semiconductor selective MM emitters with a variety of peak absorbance wavelengths for TPV applications.

Key words: Metamaterials, optical absorbers, selective emitters

\section{INTRODUCTION}

Thermophotovoltaic (TPV) systems are similar to photovoltaic systems except that they convert heat (infrared light) to electricity instead of focusing more on the visible wavelengths. The emitter, regardless of whether it is a natural material or a metamaterial (MM), in a TPV system absorbs heat via conduction, convection, and/or radiation and emits waves. According to Kirchhoff's law, ${ }^{1,2}$ at thermal equilibrium, the emissivity of an object is equal to its absorptivity. For this reason, the terms "absorption" and "emission" may be interchangeably used elsewhere in this paper unless they have to be technically distinguished. The energy conversion efficiency of a TPV diode can be improved with

(Received September 24, 2019; accepted January 24, 2020; published online February 11, 2020) a narrowband selective emitter that emits photons at just above the diode's bandgap energy as it reduces thermalization of the diode caused by photons whose energy is higher than the bandgap. ${ }^{3}$

Some metamaterials have been reported for their selective emission properties in the near- and midinfrared spectra. ${ }^{4-6}$ However, these selective MM emitters are made of multiple dielectric and metallic layers or have nanometer-scale feature sizes where fabrication is difficult and can lead to pattern imperfections that may lower the performances. Besides the difficulty of fabrication, MM emitters with feature sizes in nanometer scales may have other challenges. For example, the MM emitter's performance may be more likely to decrease with time with more materials used or more interfaces between different materials due to optical property variation or/and compound formation at elevated temperatures. This provides motivation to develop larger-feature-sized, more simply structured 
selective MM emitters with fewer different materials used. Also, the electromagnetic properties of a MM are greatly affected by the structural dimensions and refractive indices of the MM's constituent materials, and the refractive index is strongly impacted by the electrical conductivity. Compared to MMs built with dielectric and metallic materials, a semiconductor-containing MM should provide more degrees of freedom in tailoring the MM's optical properties by means of doping, which alters the electrical conductivity of the semiconductor material. Therefore, we are interested in all-semiconductor narrowband selective MM emitters with larger lateral feature sizes as deposition height can be controlled more easily in nanometer scales compared to lateral dimensions. While some other work demonstrated all-silicon MM absorbers operating at longer wavelengths, ${ }^{7-10}$ we target shorter wavelengths in the mid-IR regime for TPV applications with a higher smallest-dimension-to-peakemission-wavelength ratio than previous studies. However, the motivation of the targeted wavelength in this work should not be limited to a specific wavelength as the structure of our design can scale up/down for a variety of peak emission wavelengths for TPV applications at different temperatures.

For a plane wave that is propagating in the $x$-axis, the electric field of the wave can be represented by

$$
\vec{E}(x, t)=\overrightarrow{E_{o}}\left(e^{-k^{\prime \prime} x}\right)\left(e^{i\left(k^{\prime} x-\omega t\right)}\right)
$$

where $k^{\prime}$ and $k^{\prime \prime}$ are the real and imaginary parts of the wavenumber, respectively. The imaginary part of the wavenumber $\left(k^{\prime \prime}\right)$, which determines extinction of the wave, is represented by

$$
k^{\prime \prime}(\omega)=\frac{\omega \cdot n^{\prime \prime}(\omega)}{c}
$$

where $\omega$ is the angular frequency of the incident wave, $n^{\prime \prime}$ is the imaginary part of the material's refractive index, $c$ is the speed of light in vacuum, and frequency-dependent values are expressed with the letter " $\omega$ " between parentheses. The imaginary part of the refractive index $\left(n^{\prime \prime}\right)$ and the effective relative permittivity $\left(\varepsilon_{\mathrm{r}, \mathrm{eff}}^{\prime \prime}\right)$ are represented by

$$
\begin{gathered}
n^{\prime \prime}(\omega)=\frac{\varepsilon_{\mathrm{r}, \mathrm{eff}}^{\prime \prime}(\omega)}{2 n^{\prime}(\omega)} \text { and } \\
\varepsilon_{\mathrm{r}, \mathrm{eff}}^{\prime \prime}(\omega)=\varepsilon_{\mathrm{r}, \text { die }}^{\prime \prime}(\omega)+\frac{\sigma^{\prime}(\omega)}{\varepsilon_{o} \omega}
\end{gathered}
$$

where $n^{\prime}$ is the real part of the refractive index, $\varepsilon_{\mathrm{r} \text {,die }}^{\prime \prime}$ is the imaginary part of the dielectric relative permittivity, $\sigma^{\prime}$ is the real part of the electrical conductivity, and $\varepsilon_{o}$ is the vacuum permittivity. From the relationship between loss (absorption), electrical conductivity, and permittivity, the electrical conductivity directly relates to the loss in the material under incidence of electromagnetic waves. ${ }^{11}$ For dielectric materials, where electrical conductivity is negligible, absorption is near zero except near the wavelengths where dielectric resonance occurs, where the imaginary part of the dielectric relative permittivity $\left(\varepsilon_{\text {r,die }}^{\prime \prime}\right)$ peaks. For semiconductor materials, absorption is nonzero at many wavelengths, and from the equations above, it can be seen that absorption is dependent on the electrical conductivity, which in semiconductors, can be controlled through doping.

\section{DESIGN AND SIMULATION}

Figure 1 shows the structure and dimensions of our freestanding selective MM emitter design. The design has cylindrical features made of boron-doped $p$-Si with the diameter $D$ and height $h$. The features are arranged in the period of $p$ in both $x$ and $y$ directions. The electromagnetic responses of our MM emitter were simulated via CST Microwave Studio 2018 with a plane wave excitation at normal incidence. Due to the symmetry of the structure, the transverse electrical field (TE) and transverse magnetic field (TM) modes of polarization did not affect the results (not shown in the paper). We used tetrahedral meshes in the frequency domain solver to obtain more precise simulation for circular structures. The optical dispersion of the doped Si was represented by using the Drude dispersion model $\left(\varepsilon_{\mathrm{r}, \mathrm{eff}}(\omega)=\varepsilon_{\infty}-\frac{\omega_{\mathrm{p}}^{2}}{\omega^{2}+i \omega \gamma}\right.$, where $\varepsilon_{\infty}$ is the epsilon infinity, $\omega_{\mathrm{p}}$ is the plasma frequency, $\omega$ is the angular frequency of the incident wave, and $\gamma$ is the collision frequency). The design shown in Fig. 1 was simulated with several different values of $D, h, p$, and doping concentration of Si.

\section{RESULTS AND DISCUSSION}

Figure 2 shows the absorbance of the design with $p=2.4 \mu \mathrm{m}$ at the doping concentration of $5 \times$ $10^{19}$ atoms $/ \mathrm{cm}^{3}$ for varying $D$ and $h$ values. Absorbance $(A)$ is obtained by $A=1-R-T$ and is obviously a unitless value as reflectance $(R)$ and transmittance $(\mathrm{T})$ are the ratios between reflected/transmitted power and incident power. In Fig. 2, the wavelength at which absorbance peaks shifts to the longer wavelengths as the cylinder diameter $(D)$ or thickness of the $\mathrm{Si}(h)$ increases. At the dimensions of $D=1.6 \mu \mathrm{m}$ and $h=0.7 \mu \mathrm{m}$, the design exhibits highest absorbance of $94.8 \%$ at the wavelength of $3.47 \mu \mathrm{m}$ with the full width at half maximum (FWHM) of approximately $400 \mathrm{~nm}$. Also, in Fig. 2, we see the presence of only the electric resonant mode due to the presence of a resonant electric field. This structure does also contain a magnetic resonance outside of the frequency range of interest, and the overall structure can be considered similar to work on Huygens-type resonators for thermal emitters. ${ }^{12,13}$ Figure 3 shows simulated 

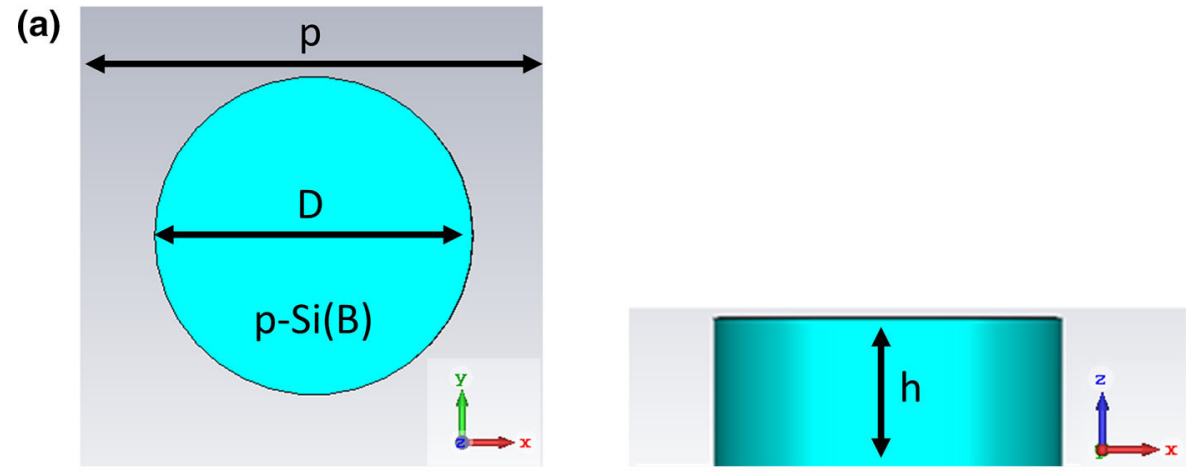

(b)
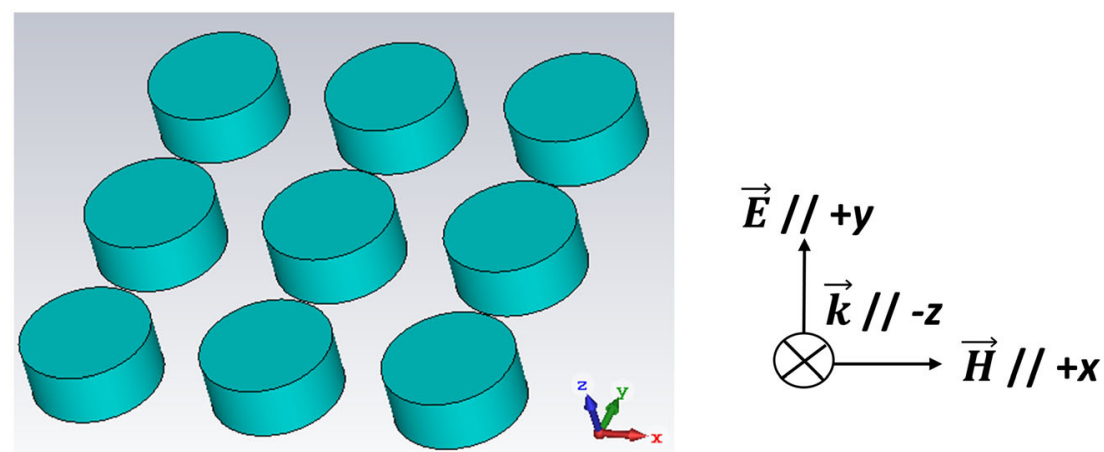

Fig. 1. Selective metamaterial emitter design. (a) Top view (left) and side view (right) of a unit cell. (b) Oblique view of several unit cells. The coordinate symbol on the bottom right corner shows the orientations of the incident electric and magnetic fields and propagation direction.

(a) $\mathrm{h}=0.6 \mu \mathrm{m}$

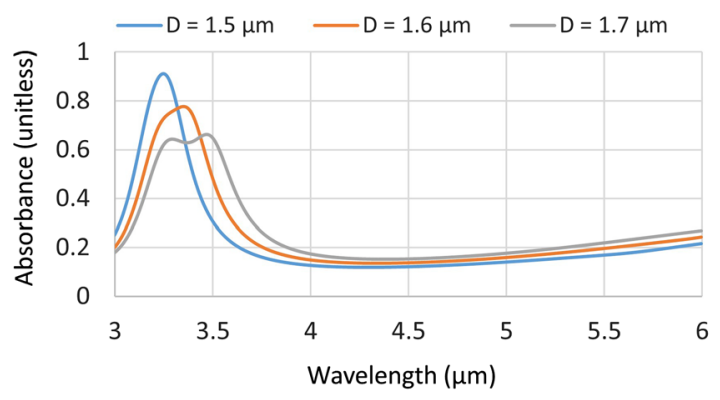

(b) $\mathrm{h}=0.7 \mu \mathrm{m}$

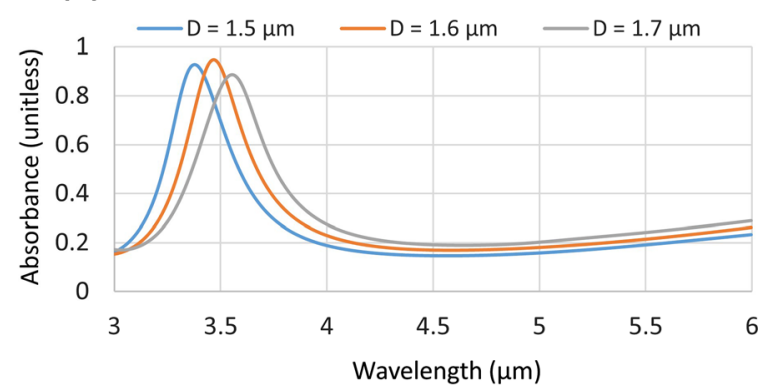

(c) $\mathrm{h}=0.8 \mu \mathrm{m}$

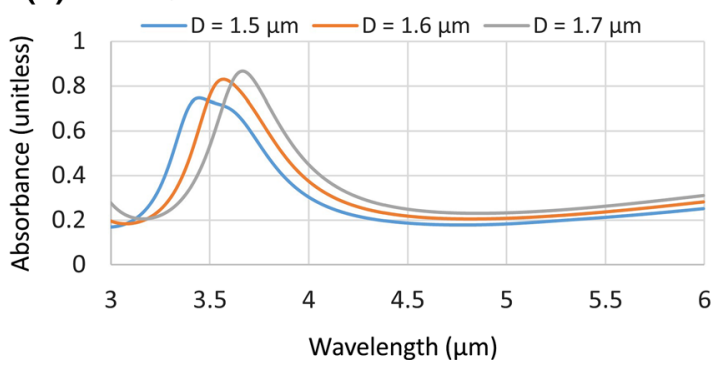

Fig. 2. Absorbance of the structure as a function of $D$ and $h$ at the doping concentration of $5 \times 10^{19}$ atoms $/ \mathrm{cm}^{3}$, where $p=2.4 \mu \mathrm{m}$. (a) $h=0.6 \mu \mathrm{m}$, (b) $h=0.7 \mu \mathrm{m}$, and (c) $h=0.8 \mu \mathrm{m}$.

absorbance of the structure with $p=2.4 \mu \mathrm{m}$, $D=1.6 \mu \mathrm{m}$, and $h=0.7 \mu \mathrm{m}$, which was the one that provided highest absorption in Fig. 2, at several different doping concentrations of Si. Table I shows the Drude dispersion parameters used for doped Si in Fig. $3 .{ }^{14}$ In Fig. 3, the peak absorbance wavelength shifts to the shorter wavelengths as the doping concentration increases, and highest 


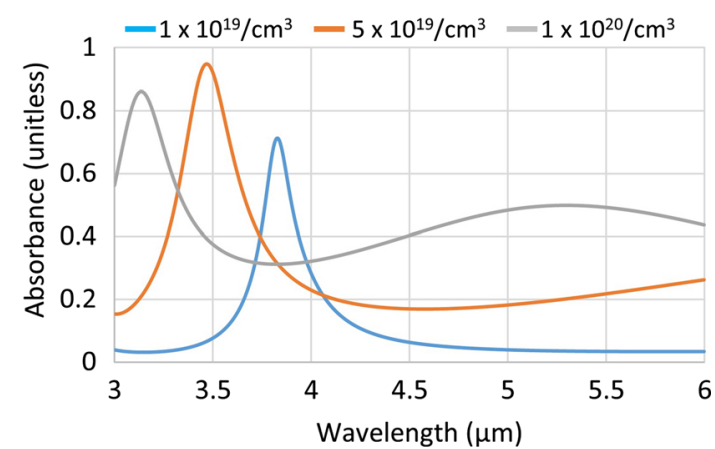

Fig. 3. Absorbance of the structure of $p=2.4 \mathrm{um}, D=1.6 \mathrm{um}$, and $h=0.7 \mathrm{um}$ at several different doping concentrations of Si. The doping concentrations are notated in the legend.

Table I. Drude dispersion parameters for doped Si in Fig. 3 (N: doping concentration)

\begin{tabular}{lccc}
\hline$N\left[1 / \mathrm{cm}^{3}\right]$ & $1 \times 10^{19}$ & $5 \times 10^{19}$ & $1 \times 10^{20}$ \\
$\varepsilon_{\infty}$ & 11.74 & 11.74 & 11.74 \\
$\omega_{p}[\mathrm{rad} / \mathrm{s}]$ & $4.356 \times 10^{14}$ & $9.741 \times 10^{14}$ & $1.378 \times 10^{15}$ \\
$\gamma[1 / \mathrm{s}]$ & $1.344 \times 10^{14}$ & $1.344 \times 10^{14}$ & $1.344 \times 10^{14}$ \\
\hline
\end{tabular}

absorption is achieved at the doping concentration of $5 \times 10^{19}$ atoms $/ \mathrm{cm}^{3}$ over the simulated wavelength range. The absorbance of the structure with $D=1.6 \mu \mathrm{m}$ and $h=0.7 \mu \mathrm{m}$ at the doping concentration of $5 \times 10^{19}$ atoms $/ \mathrm{cm}^{3}$ is also investigated for varying $p$ values to see the effects of the structure period. These results are shown in Fig. 4, where the peak absorbance wavelength shifts to the longer wavelengths and the absorbance increases as the period of the structure increases. For simulations done with a freestanding MM design, Table II shows the peak absorbance wavelengths of the structures in Fig. 4 and the required maximum refractive index of the substrate (if a substrate were used) in order to not produce diffraction. Here, the required maximum refractive index of the substrate is chosen to be the critical refractive index (real part) with which the first-order diffraction in the structure (inside the substrate) becomes evanescent at the peak absorption wavelength to maintain simulation accuracy without diffraction. From the results of Table II, we chose the structure with $p=2.4 \mu \mathrm{m}$ in Fig. 4 as the best-compromised design between absorbance and substrate choices; materials such as fused silica or Borofloat 33 glass could be candidates for the substrate for this design as their refractive indices are smaller than 1.45 at the wavelength of $3.47 \mu \mathrm{m}$. Hence, from now on we refer to the design of $p=2.4 \mu \mathrm{m}, D=1.6 \mu \mathrm{m}$, and $h=0.7 \mu \mathrm{m}$ at the doping concentration of $5 \times$ $10^{19}$ atoms $/ \mathrm{cm}^{3}$ as "the selected design". The peak absorbance wavelength of $3.47 \mu \mathrm{m}$ of the selected design corresponds to the wavelength where

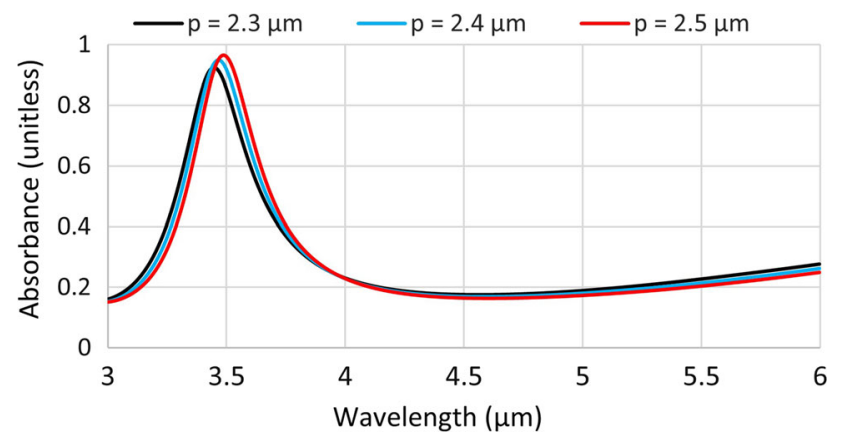

Fig. 4. Absorbance of the structure with varying values of period $(p)$, where $D=1.6 \mu \mathrm{m}, h=0.7 \mu \mathrm{m}$, and doping concentration is $5 \times$ $10^{19}$ atoms $/ \mathrm{cm}^{3}$

blackbody radiation peaks at $835 \mathrm{~K}$, which is significantly below the melting point of $\mathrm{Si}, 1687 \mathrm{~K}$. Figure 5 compares absorbance of an unpatterned $p$ $\mathrm{Si}(\mathrm{B})$ layer, a patterned $p$-Si(B) layer, and a patterned undoped Si layer. The patterned undoped Si layer, which is a pure dielectric material and therefore its electrical conductivity is near zero, is lossless over the simulated wavelengths. The unpatterned doped Si layer exhibits some absorbance higher than zero, as expected. Among the three different layers, only the patterned doped Si layer exhibits selective absorption. Figure 6 shows simulated power loss density of the selected design along with E- and H-fields at the peak absorbance wavelength. In Fig. 6(a1)-(c1) and (a2)-(c2), the magnitude distribution of the power loss density matches that of the E-field average (where the field average $\quad\langle|\vec{E}|\rangle=\frac{1}{2 \pi} \int_{0}^{2 \pi}|\vec{E}(\varphi)| \mathrm{d} \varphi, \varphi$ : phase). This implies that a significant amount of absorption occurs where the E-fields resonate in the cylinder. To further investigate the loss mechanism of the design beyond what discussed in other work where doped $\mathrm{Si}$ cylinder structures are studied, ${ }^{8,9}$ we looked into simulated vector fields. In Fig. 6(d1), the E-fields on the incident side and central part of the cylinder, marked as (1) and (2), are pointing in opposite directions, which is attributable to the slower propagation in the $\mathrm{Si}$; the real part of the Si's refractive index is 2.96 at the simulated doping concentration at the peak absorbance wavelength. The two opposing, time-varying E-fields in the figure induce an H-field pointing in the $+x$ axis in the region between the regions (1) and (2) that adds up to make a stronger H-field nearby. Similarly, in Fig. 6(e2), the H-fields in the regions (1) and (2) are pointing in opposite directions. This will induce an E-field that adds up to make a stronger E-field nearby. As a result of these mutual inductions, strong electric and magnetic fields are created within a volume in the cylinder. However, although both electric and magnetic resonances are present, a significant amount of absorption occurs where the E-fields resonate within the cylinder as discussed 
Table II. Peak absorbance wavelength, absorbance, and required maximum refractive for the structures in Fig. 4

\section{Structure period $(p)$}

Peak absorbance wavelength $\left(\lambda_{\text {peak }}\right)$

Absorbance at $\lambda_{\text {peak }}$

Required maximum refractive index $\left(\frac{\lambda_{\text {peak }}}{p}\right)$
$2.3 \mu \mathrm{m}$

$3.45 \mu \mathrm{m}$

0.924

1.5

$2.4 \mu \mathrm{m}$
$3.47 \mu \mathrm{m}$
0.948
1.45

1.45
$2.5 \mu \mathrm{m}$

$3.49 \mu \mathrm{m}$

0.966

1.40

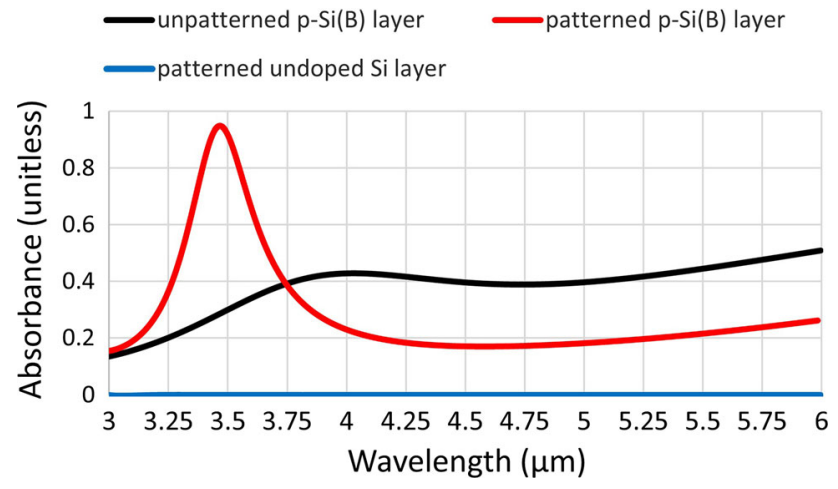

Fig. 5. Absorbance comparison of an unpatterned $p$-Si(B) layer, patterned $p-\mathrm{Si}(\mathrm{B})$ layer, and patterned undoped $\mathrm{Si}$ layer. The patterned Si layers have the dimensions of the selected design, and the doping concentration is $5 \times 10^{19} / \mathrm{cm}^{3}$ for unpatterned and patterned $p-\mathrm{Si}(\mathrm{B})$ layers

before. We interpret this as due to the fact that the material of the cylinder, $p$ - $\mathrm{Si}(\mathrm{B})$, is nonmagnetic, and therefore the physical entity that causes loss is electrons in response to electric fields. Figure 7 shows extracted effective optical properties of the selected design, assuming the MM emitter was a homogeneous material. In the extracted effective relative permittivity and permeability plots, it is seen that both optical properties exhibit resonances at near the peak absorbance wavelength, $3.47 \mu \mathrm{m}$. As the analysis of extracted optical properties gives insights to investigating the loss mechanism of the MM, ${ }^{15}$ the results of Fig. 7 agree with what we discussed before from the results of Fig. 6. Figure 8 shows absorbance of the selected design with a substrate incorporated, where the peak absorption frequency does not significantly shift. Here, the refractive index of the substrate is chosen to be 1.4 to represent fused silica or Borofloat 33 glass. However, we found that the difference between absorption spectra of the freestanding structure and on-substrate structure significantly decreases as the substrate's refractive index decreases.

\section{CONCLUSION}

The design we present here is made of a single layer of boron-doped, $p$-type $\mathrm{Si}$ cylinders. The selected design of the freestanding metamaterial emitter exhibits a peak absorbance of $94.8 \%$ at the wavelength of $3.47 \mu \mathrm{m}$ (83.3\% of absorption at the wavelength of $3.57 \mu \mathrm{m}$ when a substrate with the refractive index of 1.4 is used). The high absorption of the design is realized due to the resonances of electric and magnetic fields in the Si cylinder. The smallest dimension of the selected design in this study is $0.8 \mu \mathrm{m}$. This can be resolved by an advanced photolithography tool and does not require electron beam lithography, which takes a significantly longer time to generate patterns over the substrate. Therefore, we believe that our work demonstrates a path for future research on largerarea all-semiconductor narrowband selective MM emitters operating at shorter wavelengths for TPV applications. 
(a1) Power loss density

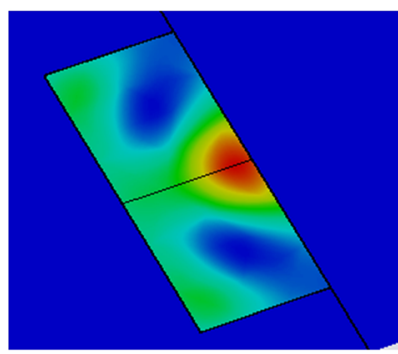

(d1) E-field vectors

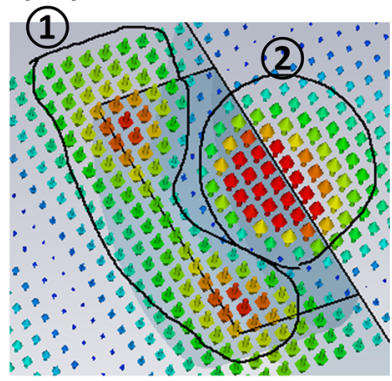

(a2) Power loss density

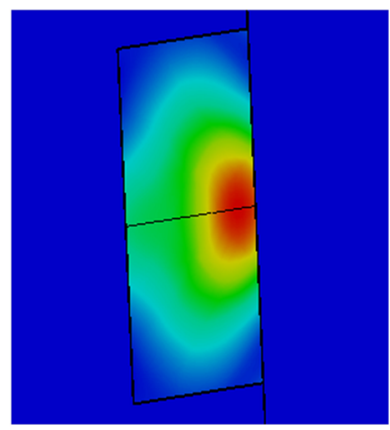

(d2) E-field vectors

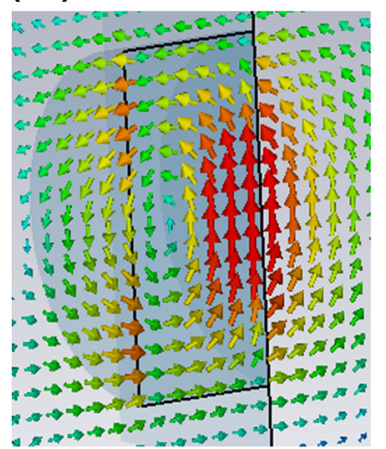

(b1) E-field average

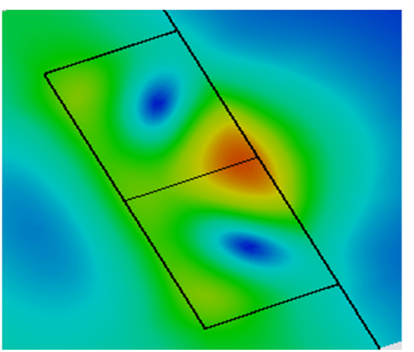

(e1) $\mathrm{H}$-field vectors

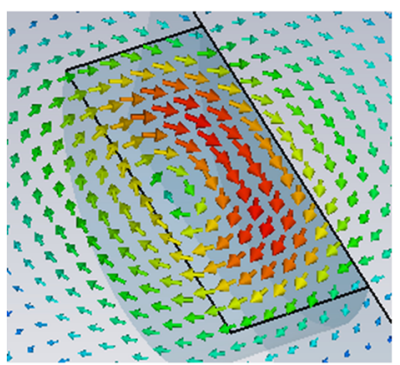

(c1) $\mathrm{H}$-field average
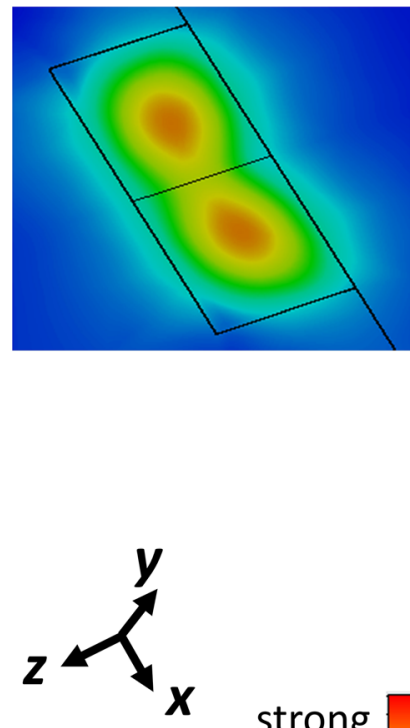

strong (b2) E-field average

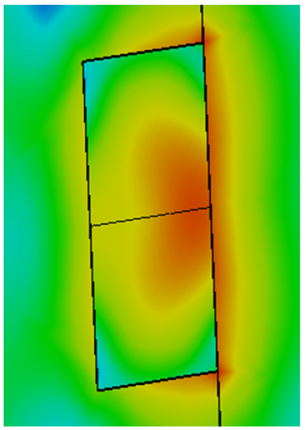

(e2) $\mathrm{H}$-field vectors

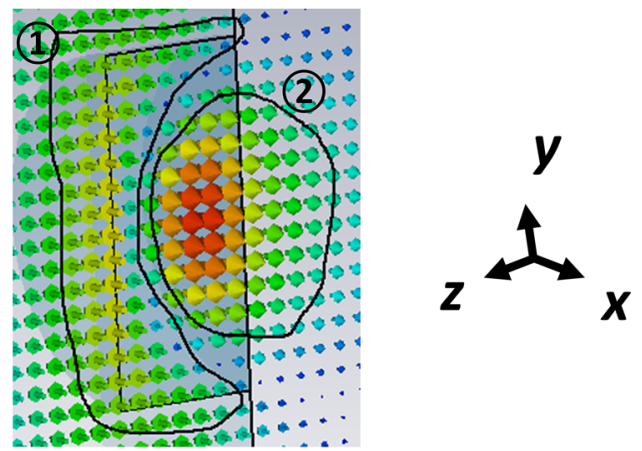

Fig. 6. Snapshots of simulated fields of the selected design on different planes at the same time at the peak absorbance wavelength; $3.47 \mu \mathrm{m}$. (a1)-(e1) Fields on the $x z$ plane. (a2)-(e2) Fields on the $y z$ plane. (a1) and (a2) Power loss density. (b1) and (b2) E-field average. (c1) and (c2) $\mathrm{H}$-field average. (d1) and (d2) E-field vectors. (e1) and (e2) $\mathrm{H}$-field vectors. The relative intensities of the fields are represented by color. 
(a) Relative permittivity

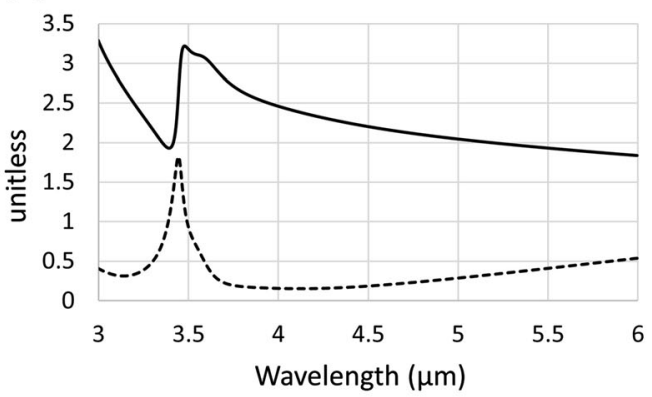

(c) Refractive index

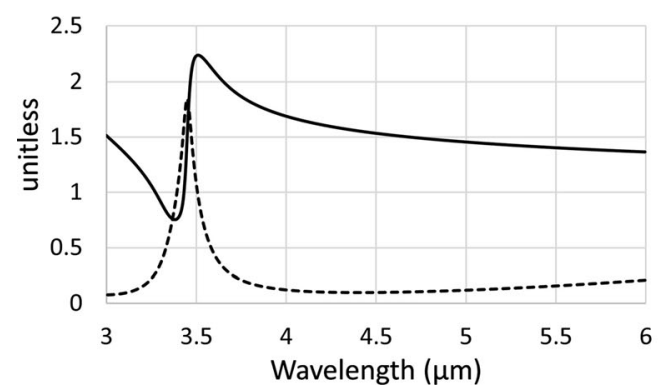

(b) Relative permeability

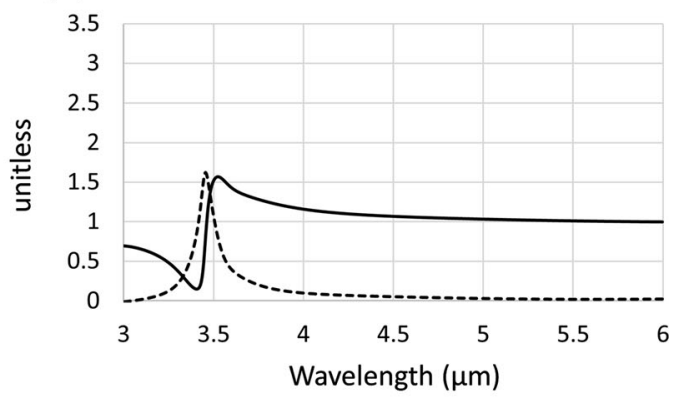

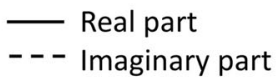

Fig. 7. Extracted effective optical properties of the selected MM emitter design. (a) Extracted effective relative permittivity. (b) Extracted effective relative permeability. (c) Extracted effective refractive index.

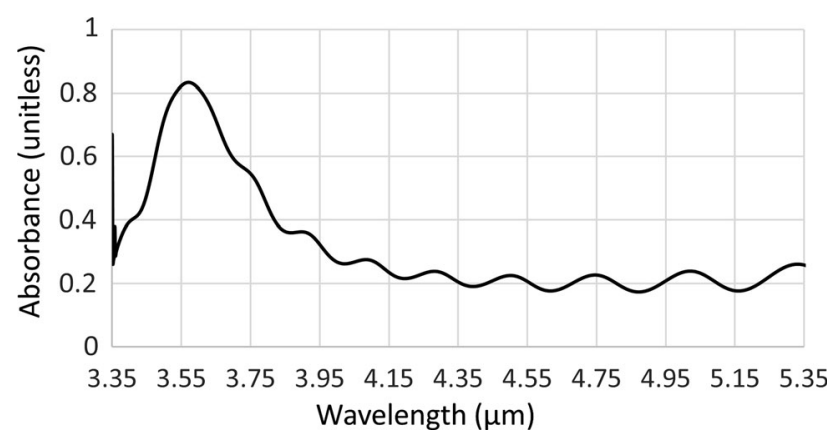

Fig. 8. Absorbance of the selected design coupled with a substrate, $30 \mu \mathrm{m}$ thick. The effect of substrate thickness is found to be negligible on the absorbance spectrum.

\section{ACKNOWLEDGMENTS}

Funding was provided by the Office of Naval Research (Grant No. N00014-15-1-2946), National Science Foundation (Grant No. ECCS - 1806311).

\section{OPEN ACCESS}

This article is licensed under a Creative Commons Attribution 4.0 International License, which permits use, sharing, adaptation, distribution and reproduction in any medium or format, as long as you give appropriate credit to the original author(s) and the source, provide a link to the Creative Commons licence, and indicate if changes were made. The images or other third party material in this article are included in the article's Creative Commons licence, unless indicated otherwise in a credit line to the material. If material is not included in the article's Creative Commons licence and your intended use is not permitted by statutory regulation or exceeds the permitted use, you will need to obtain permission directly from the copyright holder. To view a copy of this licence, visit http://creativecom mons.org/licenses/by/4.0/.

\section{REFERENCES}

1. T. Inoue, M. De Zoysa, T. Asano, and S. Noda, Optica 2, 27 (2015).

2. C. Shemelya, D. DeMeo, N.P. Latham, X. Wu, C. Bingham, W. Padilla, and T.E. Vandervelde, Appl. Phys. Lett. 104, 201113 (2014).

3. N.A. Pfiester and T.E. Vandervelde, Phys. Stat. Sol. (A) 214, 1 (2017).

4. F.T. Maremi, N. Lee, G. Choi, T. Kim, and H.H. Cho, Energies 11, 1 (2018).

5. H. Wang and L. Wang, Opt. Expr. 21, A1078 (2013).

6. X. Han, K. He, Z. He, and Z. Zhang, Opt. Expr. 25, A1072 (2017).

7. K. Gorgulu, A. Gok, M. Yilmaz, K. Topalli, N. Blylkll, and A.K. Okyay, Sci. Rep. 6, 1 (2016).

8. X. Liu, K. Fan, I.V. Shadrivov, and W.J. Padilla, Opt. Expr. 25, 191 (2017).

9. K. Fan, J.Y. Suen, X. Liu, and W.J. Padilla, Optica 4, 601 (2017).

10. C. Wu, N. Arju, G. Kelp, J.A. Fan, J. Dominguez, E. Gonzales, E. Tutuc, I. Brener, and G. Shvets, Nat. Commun. 5 , 3892 (2014).

11. C. Bishop, The Relationship Between Loss, Conductivity, and Dielectric Constant. http://pleclair.ua.edu/impedance/The $\% 20$ Relationship\%20Between\%20Loss.pdf. Accessed 12 Aug 2019.

12. A. Howes, J.R. Nolen, J.D. Caldwell, and J. Valentine, $A d v$. Opt. Mater (2019). https://doi.org/10.1002/adom.201901470. 
Design of an All-Semiconductor Selective Metamaterial Emitter in the Mid-IR Regime with Larger Feature Sizes for Thermophotovoltaic Energy Conversion Applications

13. S. Campione, L.I. Basilio, L.K. Warne, and M.B. Sinclair, Opt. Express 23, 2293 (2015).

14. T.E. Tiwald, ETD Collection for University of Nebraska Lincoln (1999).

15. C.M. Watts, X. Liu, and W.J. Padilla, Adv. Mater. 24, OP181 (2012).
Publisher's Note Springer Nature remains neutral with regard to jurisdictional claims in published maps and institutional affiliations. 